\title{
Subdural empyema: A review of 29 cases
}

\author{
EDWARD HITCHCOCK ${ }^{1}$ AND ALEXANDER ANDREADIS ${ }^{2}$ \\ From the Department of Neurological Surgery, Radcliffe Infirmary, Oxford
}

In 1948, Schiller, Cairns, and Russell presented 33 cases of subdural empyema arising over 20 years most of which were treated in this department. Their paper dealt with the results of penicillin instillations into the subdural space and marked a major therapeutic advance in the treatment of this condition. We felt it would be of interest to review 29 subsequent cases and to note the present-day problems in their management.

The history of the recognition of this condition has been outlined in detail by Kubik and Adams (1943) and by Courville (1944), and there is little to add to their accounts.

Schiller et al. (1948) used the term 'purulent pachy meningitis interna' because of a supposed resemblance to peritonitis. 'Subdural abscess' is often used, but should only be applied when there is loculation and a membrane is formed. Subdural empyema is a better term, indicating pus in a pre-formed space.

\section{AETIOLOGY}

Stern and Boldrey (1952) listed 10 aetiological conditions for the production of this condition, but the commonest cause of subdural empyema is otorrhinological disease. In our series of 29 cases 26 arose as complications of ear or nose infections whilst Schiller et al. (1948) reported 29 such cases out of a total of 33.

Much less commonly it follows the rupture of an intracerebral abscess, and rarely it is secondary to penetrating wounds of the skull and cranial osteomyelitis from other causes. Other less common causes are leptomeningitis and bacteraemia, or infection of subdural haematoma (Stern and Boldrey, 1952). Of the three 'miscellaneous' cases in our series one followed ventriculography, one was presumably an infected subdural haematoma in a child, and the remaining patient developed a pre-pontine subdural empyema secondary to osteomyelitis of the skull base.

Early reports of subdural infection usually referred to otitic disease as the cause but from the late 1930s

1Present address: The Royal Infirmary, Manchester.

${ }^{2}$ Present address: Polyclinic of Athens, 3 Piraeus Street, Athens, Greece. sinus infection became increasingly responsible, an today sinus infection far exceeds otitic as the cause of subdural empyema. Thus in our series 20 cases were 'paranasal' and six 'otitic' whereas Schiller et ale (1948) had 23 cases following sinus disease and sixp cases from mastoid infection. The reason is not im 2 mediately apparent. Probably many patients suffere屯্ in the past from undiagnosed sinus infection.

Stern and Boldrey (1952) and others have noted $a$ relative increase in the incidence of subdurat empyema as compared to brain abscess. Schille? et al. (1948) reported 33 cases of subdural empyem in 20 years. Since then in this department we have had 31 cases (two are excluded from this series be cause of insufficient information) in the 14 years from 1949 to 1962 and the number of cases of briin abscess admitted during this time was 115 . $\mathbb{S}_{\mathrm{O}}$ fortunately we are unable to quote comparaiple numbers of abscesses for the 20 years covered in paper of Schiller et al. (1948) as some of their cases of subdural empyema were treated elsewhere. Hogvo ever, by dividing the last 14 years into two equat periods some conclusions can be drawn. There wer 69 cases of brain abscess and 14 of subdural empyem $\$$ during the years 1949 to 1955 and 46 of brain absces and 17 of subdural empyema during the second seven year period. Although the numbers are small they appear to support the observation of Stern and Boldrey (1952).

Table I outlines the cases of this series groupe $\mathbb{P}$ under age, sex, and primary focus of infection. There is a preponderance of males over females, 23 to six and this has been noted by many authors, although no explanation can be given. It cannot be related te. the larger size of the paranasal sinuses in men, sinces the condition is common in children when the sinuse are hardly formed, and neither does it appear t bear any relation to occupation. The age ranged from. 8 months to 67 years, but the overall peak age lap between 10 and 20 years. In otitic disease, howeve? the peak lay in the age group 40 to 60 years.

The incidence of acute or chronic disease was different for paranasal or otitic infections (see Table I) In the 'paranasal' group 17 patients had acute in fections without a history of chronic sinusitis an $\$$ only three patients had chronic infections. Cons 
TABLE I

AGE, SEX, AND ORIGIN OF THE 29 CASES

\begin{tabular}{|c|c|c|c|c|c|c|}
\hline & \multicolumn{2}{|c|}{ Age (years) } & \multirow[b]{2}{*}{$20-40$} & \multirow{2}{*}{$40-60$} & \multirow[b]{2}{*}{$60+$} & \multirow[b]{2}{*}{ Tota } \\
\hline & $0-10$ & $10-20$ & & & & \\
\hline Male & 3 & 8 & 6 & 4 & 2 & 23 \\
\hline Female & $\mathbf{0}$ & 3 & 0 & 2 & 1 & 6 \\
\hline Total & 3 & 11 & 6 & 6 & 3 & 29 \\
\hline Died & 1 & 4 & 0 & 3 & 2 & 10 \\
\hline \multicolumn{7}{|l|}{ Paranasal } \\
\hline Chronic acute & 0 & 0 & 3 & 0 & $\mathbf{0}$ & 3 \\
\hline Acute & 2 & 10 & 3 & 2 & $\mathbf{0}$ & 17 \\
\hline Total & 2 & 10 & 6 & 2 & $\mathbf{0}$ & 20 \\
\hline Died & 0 & 3 & $\mathbf{0}$ & 1 & 0 & 4 \\
\hline \multicolumn{7}{|l|}{ Otitic } \\
\hline Chronic acute & 0 & 1 & $\mathbf{0}$ & 2 & 2 & 5 \\
\hline Acute & 0 & 0 & 0 & 1 & 0 & 1 \\
\hline Total & 0 & 1 & 0 & 3 & 2 & 6 \\
\hline Died & 0 & 1 & 0 & 1 & 1 & 3 \\
\hline \multicolumn{7}{|l|}{ Others } \\
\hline Total & 1 & 0 & 0 & 1 & 1 & 3 \\
\hline Died & 1 & $\mathbf{0}$ & 0 & 1 & 1 & 3 \\
\hline
\end{tabular}

versely with the 'otitic' group only one patient had acute otitis, the remaining five presenting with acute exacerbations of chronic ear infections. The numbers in the otitic group were small, but these findings may be related to the different modes of spread of infection.

Seven patients had had operations on the ears or nose before admission to this department. In five of them signs of subdural involvement were already present. It seems very unlikely, therefore, that such procedures cause subdural infection.

\section{PATHOLOGY}

Since the brain and membranes fill the cranium completely the subdural space is a potential space. The dorso-lateral part, however, together with the interhemispherical fissure, is relatively free, imposing no obstruction to the formation and flow of fluid (Schiller et al., 1948). The 'basal' subdural space is practically non-existent, and fluid accumulations at this site are therefore uncommon and small (Courville, 1944).

Infection may enter the subdural space by two routes:

1 Direct extension by erosion of osteitic areas of the bony wall and of the dura separating the purulent focus from the subdural space: according to Courville (1944) this is uncommon in paranasal sinus infections. In otitic infections, however, this route seems more common; in our six cases four had dural involvement observed either at necropsy (two cases) or at operation (two cases). In the remaining two cases the information was inadequate. This observation may account for the preponderance of chronic infections in the otitic group.
2 Indirect extension is more common in paranasal sinus disease. According to Courville (1944) the mode of spread is by progressive thrombophlebitis of the mucosal veins and subsequent spread to dural veins, venous sinuses, and cerebral veins. Orbital oedema indicates extension of thrombophlebitis along the veins of the orbit and it is frequently found. Indeed, Stevens and Welch (1956) reported retinal thrombophlebitis in one case. The infection may limit itself to the subdural space, or to the brain, producing either a subdural empyema or an intracerebral abscess following cortical thrombophlebitis. Not infrequently subdural empyema and intracerebral abscess may thus arise simultaneously.

Entering the subdural space the infection elicits a prompt inflammatory reaction with exudate. Pus is rapidly formed and membrane formation, although it does occur, is not marked in the early stages. Wood (1952) stated that antibiotics may allow time for membrane and granulation tissue to form but in our series it was encountered only twice at the first operation.

The extension of pus depends on the primary site of infection and the anatomical peculiarities of the subdural space. Collections beneath the hemispheres are uncommon and thin, and dorso-lateral and interhemispherical (Figs. 1 and 2) collections common (Courville, 1944). Because these desperately ill patients lie recumbent gravity aids spread posteriorly. From the study of our cases, both at operation and necropsy, the extension of pus from the various primary sites appeared to follow fairly well-defined paths.

PARANASAL SUPPURATION Since the portal of entry is usually through communicating veins, subdural collections usually form at the frontal poles in relation to the affected sinus. In the fronto-polar route the pus extends posteriorly over the convexity of the frontal lobe. This occurred in 19 of our 20 'paranasal' cases. The spread was diffuse with a tendency to form the major collection over the dorso-lateral aspect of the hemisphere (Fig. 3). Its posterior limit was variable. In five patients the collection was limited to the frontal lobe, in 10 patients it had reached the mid-parietal region, and in the remaining four it extended to the parieto-occipital area. None appeared to have reached the convexity of the occipital pole. Pus extended along the Sylvian fissure in eight patients. In four patients pus was found alongside the falx, presumably having seeped between the superior cerebral veins and pacchionian granulations.

The parafalcine route may allow the spread of the pus either posteriorly or beneath the free edge of the falx to the opposite hemisphere. The latter occurred 


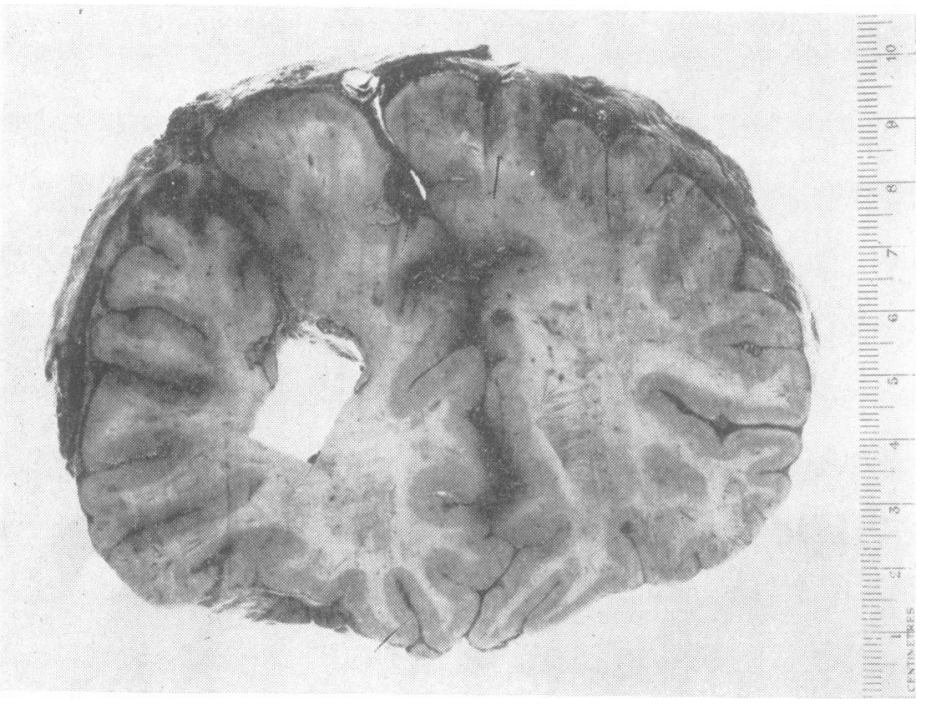

FIG. 1. Coronal section of the brain from a case of subdural empyema showing pus over the dorso-lateral aspect of the hemisphere.

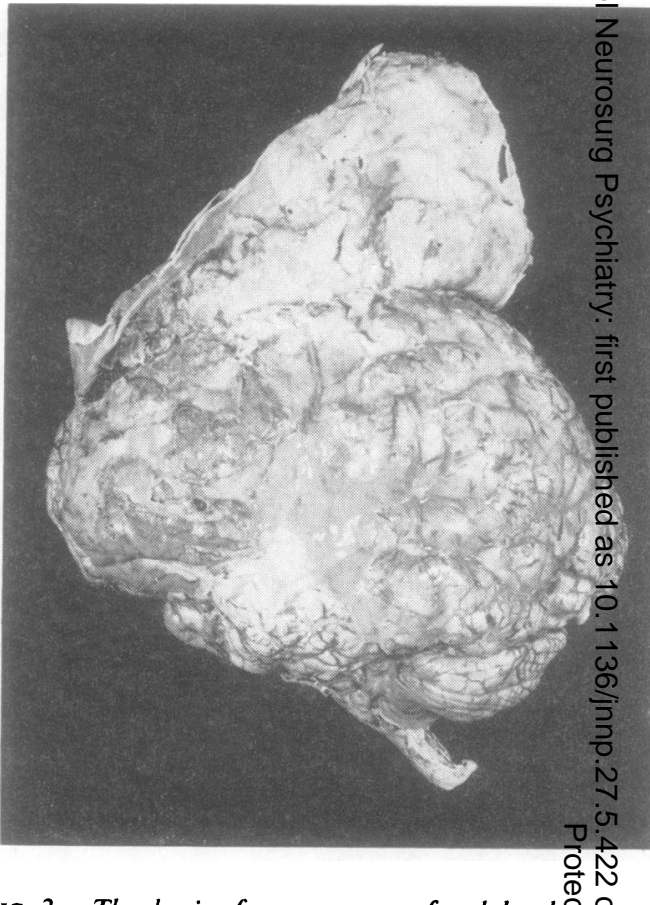

FIG. 3. The brain from a case of subdural @eppe showing the extent of the collection of pusaover dorso-lateral aspect of the hemisphere.

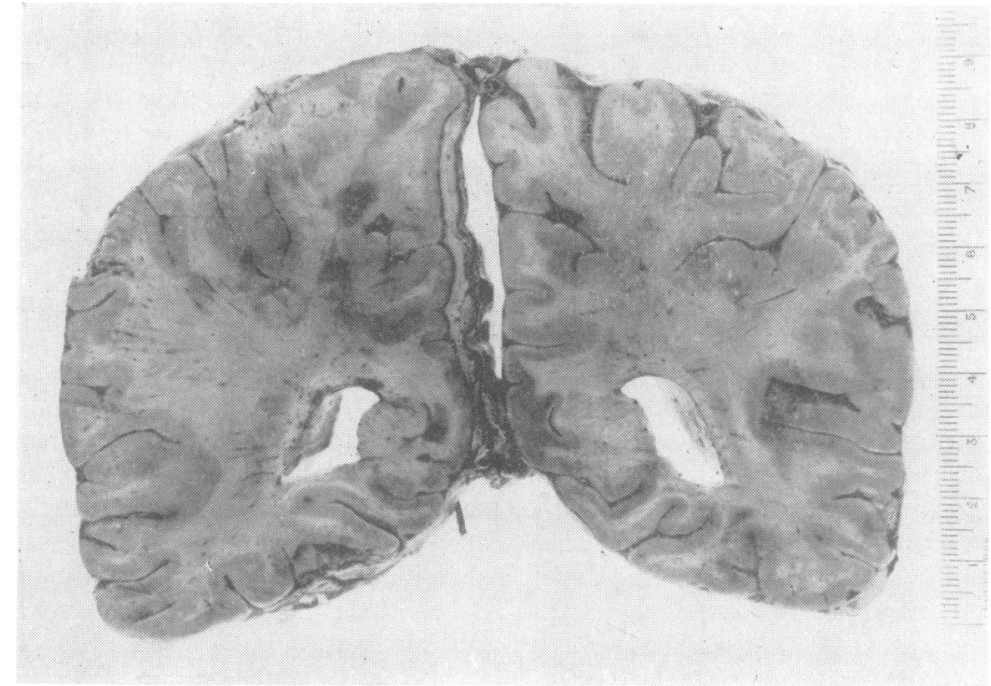

FIG. 2. Coronal section of the brain from a case of subdural empyema showing collection of pus on the medial surface of the brain alongside the falx. 
in three of the four patients with secondary falcine collections and these three patients are the only 'paranasal' cases with bilateral subdural empyema. Primary falcine collection occurred once only.

Subfrontal collections were found in three patients, in one at operation and in the remaining two at necropsy.

OTITIC SUPPURATION Intracerebral abscess is much commoner than subdural empyema in this condition. When a subdural collection does form, however, it tends to pass posteriorly and medially over the tentorium towards the falx, and extend along the routes already outlined. In its course pus may seep over the edge of the tent into the posterior fossa and then a cerebellar subdural empyema results. More commonly, however, the pus remains above the tentorium. Its progress along the falx is impeded by gravity and it rarely extends far anteriorly. Direct extension over the lateral aspect of the temporal lobe is less common. Three of our six otitic cases had clear evidence of pus along the falx and/or the occipital poles, and in these no pus was found over the temporal lobe. In another two cases pus was distributed over the major part of the convexity, but in one case the presence of a falcine collection could not be excluded. In the remaining case the pus was confined to the posterior fossa obviously through direct spread of the infection from the petrous bone.

To sum up, there are three common sites for pus to collect in subdural empyema, namely, the frontal poles extending on to the fronto-parietal regions, the para-falcine spaces, and the occipital poles. This information is essential to the correct placing of burr holes, and collections at these sites deserve consideration as causes of relapse.

The following summary illustrates the spread of pus along the falx and in this case into the posterior fossa. It also emphasizes that intracerebral abscess or intracerebellar abscess should be considered in patients failing to improve or relapsing.

CASE 1 B.P. (R.I. 104616), a 13-year-old boy, suffered from bilateral chronic otitis media and had had a right radical mastoidectomy five years earlier. He was admitted with a one-month history of vertigo and increasingly severe headache following an upper respiratory tract infection. The discharge from the left ear became profuse and finally he was admitted to another hospital on 18 April, 1949 with signs of meningitis. His cerebrospinal fluid contained 1,800 cells per c.mm., mostly polymorphs, and he was treated with intramuscular penicillin, one dose of intrathecal penicillin, and oral sulphadiazine. Within two days the cerebrospinal fluid had improved, but his general condition had deteriorated and he became comatose with a right hemiparesis. A left radical mastoidectomy was performed immediately and revealed a large extradural abscess and granulations over the middle fossa dura. The following day, 21 April, he had a complete right hemiplegia with right-sided convulsions and remained comatose. The left temporal lobe was explored through the mastoidectomy wound but no abscess found. He gradually improved, however, over the next two weeks but on 5 May was drowsy with a pyrexia of $103^{\circ} \mathrm{F}$. and was transferred to this department. Here he was found to be very drowsy with mild bilateral papilloedema and a right hemiparesis. A diagnosis of left temporal lobe abscess was made and a left temporal burr hole was made immediately. Needling of the temporal lobe revealed no abscess but a left frontal burr hole allowed the evacuation of about $20 \mathrm{ml}$. of thick subdural pus from the medial side of the hemisphere. A catheter was inserted $5 \mathrm{~cm}$. along the falx directed posteriorly for penicillin instillations. During the operation there was a dramatic improvement and within 24 hours he was eating and sitting up in bed. Regular instillations of penicillin down the tube had been continued as well as intramuscular penicillin and oral sulphadiazine. Two days after the first operation, however, (7 May) the level of consciousness deteriorated and a further operation was performed to exclude a posterior loculation. A left occipital burr hole, later enlarged, demonstrated about $20 \mathrm{ml}$. of thick pus along the medial side of the hemisphere. A catheter was inserted along the falx and the left falcine subdural space irrigated with penicillin between the frontal and occipital catheters. Again there was a dramatic improvement. On 10 May, the initial subdural pus was reported as growing proteus organisms and streptomycin was now administered, both subdurally and systemically. About this time he was noted to have attacks of titubation and left-sideu tremor, and the following day (11 May) respiration suddenly ceased and he died.

Necropsy revealed a left frontal lobe convexity subdural empyema and a large abscess in the left para-falcine subdural space extending from the frontal to the occipital pole. There was an extensive subdural empyema over the superior surface of the cerebellum and a large left cerebellar abscess associated with a dural slough overlying an osteitis of the left petrous bone.

\section{BACTERIOLOGY}

The commonest organism isolated was streptococcus, in half of the cases in its anaerobic form. Other organisms (Staphylococcus and Gram-negative bacilli such as Clostridium and especially Proteus) also occurred. Table II shows the numbers actually grown. In two patients no bacteriology was done and in another four no growth was obtained, although in two of these four patients Gram-positive cocci were seen on films.

Schiller et al. (1948) had positive cultures in 27 patients and in 24 of these streptococci, presumably sensitive to penicillin, were grown. Even supposing that in the remaining three cases the organisms were penicillin-resistant, comparison with our figures shows that penicillin-resistant organisms are in- 
TABLE II

ORGANISMS IN SUBDURAL EMPYEMA

Organisms on Culture Number of Penicillin- Penicillin- Sensitivity Patients sensitive resistant Unkown

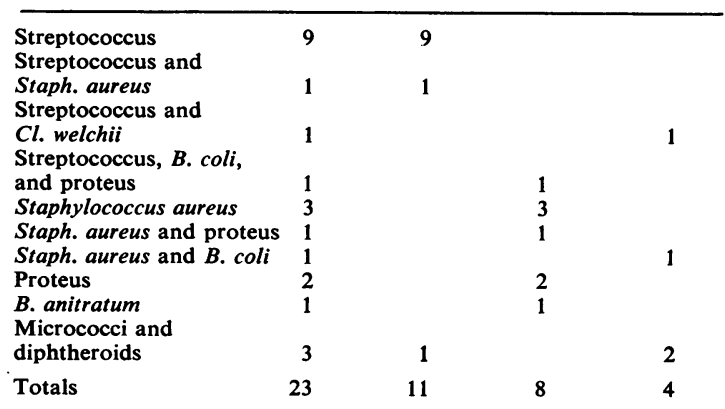

creasingly grown in these infections. This is particularly evident in the 'otitic' group where four of our six patients had proteus.

\section{ASSOCIATED LESIONS}

Their relative frequency appears to vary from author to author. Swelling of the scalp was grossly evident in four of our cases, and was then associated with osteomyelitis or extradural abscess as in Potts 'puffy tumour'. In two cases there was frank cellulitis with brawny induration and fluctuation due to subgaleal pus. In one case where a scalp flap was raised the galea and pericranium were necrotic with pus oozing through in many places. Neither of these cases had peri-orbital oedema, and this supported Courville's view that the lesions are concomitant rather than consecutive.

Whilst some authors have found osteomyelitis or extradural abscess in all their cases, others have not. Courville (1944) regarded them as uncommon accompaniments of subdural empyema, and conversely stated that in cases of osteomyelitis of the skull subdural suppuration is uncommon. In our series, frank osteomyelitis occurred in $10(50 \%)$ of the paranasal cases and in three $(50 \%)$ of the otitic. The bone in these cases of acute infection was still firm, although pus would often well into the burr holes from the diploe.

Lepto-meningeal reactions, as judged by pleocytosis in the cerebrospinal fluid, were relatively common in our experience. Eight of the nine cases coming to necropsy had fibrinous exudate in the subarachnoid space under the subdural empyema or small subarachnoidal purulent collections.

It is perhaps remarkable that the cortex escapes direct invasion for so long, separated as it is from large collections of subdural pus by the thin layer of cells forming the arachnoid. This tenuous membrane is known to be relatively impermeable to toxins and pus, and certainly if it were not, we could not expect? any of the complete recoveries from subdura empyema that now commonly occur. The barrier is penetrated at many points, especially at the vertex by cerebral veins as they enter the dural sinuses. Inêे fection may enter the cortex by an extending throm $=$ bophlebitis and result in localized cortical or subs cortical inflammation. Five cases had intracerebra? abscess. Characteristically, many of these were mul tiple and small, but tended to coalesce and form onelarge single cavity often of considerable extent.

\section{CLINICAL FEATURES}

The clinical presentation of this condition is variable but certain general features are common. The tem perature rises and there is severe generalized head $\$$ ache. Vomiting is common. The patient gradually or rapidly becomes irritable and drowsy, but these changes are often insidious and to the inexperienced appear only as a vague generalized deteriorations frequently attributed to the primary infection, as at this stage there may be little in the way of focah neurological abnormalities. Meningism is common and indeed the cerebrospinal fluid may contain a hight concentration of protein and moderate pleocytosis $N$ In the early stages the cerebrospinal fluid is sonf르은 times normal, however, and such a finding may courage the view that the central nervous system unaffected. This period of invasion usually extenglsô from 24 to 36 hours. Less commonly the diseasegis fulminating in character and symptoms of invasige of the subdural space quickly follow symptoms of theos primary infection. These cases are clearly those of ${ }^{+}$ overwhelming infections and the history may be as short as six or seven hours.

Finally, focal disturbances appear. In dominant hemisphere collections, dysphasia is a frequent earlyo symptom, often appearing as a reluctance to talk and ${ }_{\Omega}^{\Phi}$ regarded by the inexperienced as an expected ac- $\vec{F}$ companiment to the headache and systemic infec-을 tion. Minimal weakness of one or more limbs pro-? gresses insidiously or rapidly to complete paralysis.0 In some cases this is preceded by focal or genera epileptic seizures. Papilloedema may be noted. It is usually not until this stage is reached that the diag-:nosis is considered and rarely is there any delay in 3 transferring patients to a neurosurgical unit for assessment although almost invariably with a diagnosis of intracerebral abscess. By now, the patient is stuporous or frankly comatose and if transfer is delayed the 3 condition progresses to a moribund state with de $-\frac{7}{0}$ cerebrate rigidity. The time taken by the infection to manifest itself eloquently by the occurrence of $N$ focal fits, hemiplegia, aphasia, stupor, and so forth is variable. It may be as short as a few hours or as 
long as 11 days from the stage of invasion, as in one of our patients.

Table III presents the relative frequency of symptoms and signs in our cases. It should be noted that headache, present in $100 \%$ in the early stages, is reduced to $75 \%$ in the established stage and this apparent improvement is really a reflection of the deterioration of consciousness. It should also be noted that the relative frequency of symptoms in the paranasal group closely parallels that in the otitic cases. Patients with pus on the convexity of the brain usually present with contralateral hemiplegia, often with focal seizures of face, arm, and leg. Sensory disturbances are less common, whilst in collections over the dominant hemisphere aphasia is usual. The single primary falcine collection in our series presented with a lower limb monoplegia which rapidly progressed up the trunk to involve the arm, but characteristically spared the face. It differs in no way from secondary falcine collections.

TABLE III

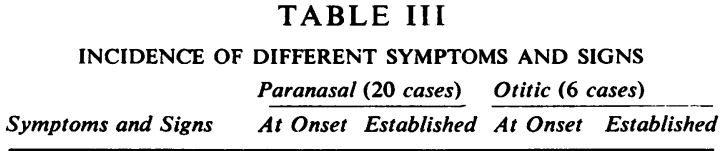

\section{Evidence of systemic}

Evidence of systemic
infection
Paralysis (usually hemi-
plegia)

Stiff neck and Kernigism

Disturbed consciousness

Headache

High intracranial pressure

Mental changes

Vomiting

Epilepsy

Visual field defects

Sensory changes

Aphasia (in left hemi-

sphere involvement)

$\begin{array}{rlll}19 & 20(100 \%) & 5 & 6(100 \%) \\ 13 & 20(100 \%) & 4 & 6(100 \%) \\ 12 & 18(90 \%) & 5 & 6(100 \%) \\ 13 & 16(80 \%) & 5 & 6(100 \%) \\ 20 & 15(75 \%) & 4 & 4(66 \%) \\ 8 & 15(75 \%) & 2 & 3(50 \%) \\ 10 & 12(60 \%) & 3 & 4(66 \%) \\ 10 & 10(50 \%) & 4 & 4(66 \%) \\ 7 & 9(45 \%) & 2 & 3(50 \%) \\ 1 & 9(45 \%) & 2 & 3(50 \%) \\ 5 & 8(40 \%) & 1 & 4(66 \%) \\ & 100 \% & & 80 \%\end{array}$

It has been stated (Schiller et al., 1948) that adequate treatment with penicillin may modify the clinical picture. Unfortunately information about the antibiotics given for the primary infection before admission to this department was not adquate enough to permit analysis. In the few cases where information was available the antibiotics did not appear to influence either the time of onset of the subdural empyema or its subsequent course. In only two cases was there transient relief of early symptoms with subsequent relapse. Furthermore the clinical picture as described by Schiller et al. (1948) differs in no way from that in our series.

\section{SPECIAL INVESTIGATIONS}

Although the diagnosis may often be made on clinical grounds, special investigations are often needed to establish it definitely. Because of this a critical examination has been made of the various special investigations used in the diagnosis of the condition.

Table IV represents the findings for cerebrospinal fluid in cases where this investigation was performed (the three untreated cases have been excluded). High protein concentrations and pleocytosis are common but eight cases $(35 \%)$ presented with nearly normal cerebrospinal fluid and with only slight pleocytosis or raised protein level. One case at the onset presented with a relatively low protein and a lymphocytic pleocytosis. As a result of surgical treatment there was in most cases a rapid fall in the cell count and usually in the protein content although the latter sometimes rose despite clinical improvement. In one case only were organisms found in the cerebrospinal fluid, illustrating the rarity of frank meningitis.

\section{TABLE IV}

\section{CEREBROSPINAL FLUID}

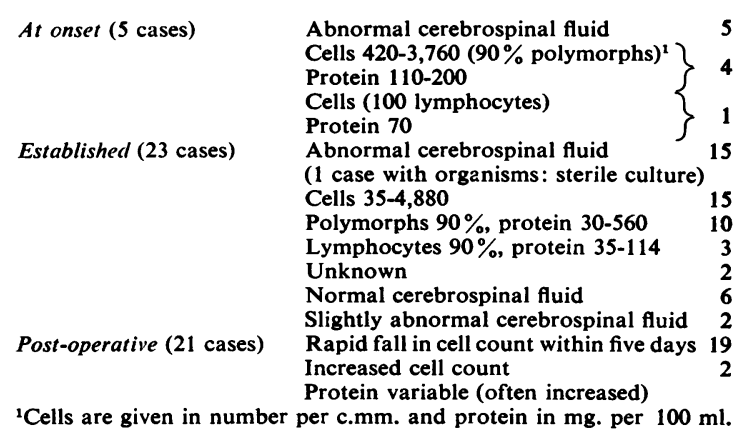

A plain skull radiograph may yield valuable information. In the 'paranasal' group 19 out of 20 cases had plain skull radiographs taken, and 18 of these showed opacity of the frontal and ethmoidal sinuses, often as part of a pansinusitis, which was, however, always predominantly unilateral and on the same side as the intracranial collection. Three cases showed evidence of early osteomyelitis of the sinus wall. Two 5-year-olds had well-marked suture diastasis, and in one other case there was a pineal shift. Only one case out of the 19 had a completely normal skull radiograph. All six otitic cases had skull radiographs taken and all showed evidence of mastoid disease on the appropriate side, manifest by loss of cellularity, sclerosis, or erosion. One case demonstrated a well-marked pineal shift. Only two of the three miscellaneous cases had skull radiographs taken; in an 8-month-old child with pus on the convexity there was progressive increase in skull size with suture diastasis, and in the remaining case 
retrospective examination of the radiographs revealed osteomyelitis of the dorsum sellae. While it must be acknowledged that many of these changes are not-specific, it is noteworthy that evidence of sinus or mastoid disease was present in the vast majority of cases and always this was most marked on the same side as the intracranial collection.

Eighteen cases had electroencephalographic recordings pre-operatively, post-operatively, or both. Ten cases had pre-operative E.E.G.s, and here the usual findings were lateralized, frequently focal, slow waves and sometimes suppression of activity over the affected area. The focal slow waves often encouraged the diagnosis of intracerebral abscess. In eight out of the 10 cases the localization was fairly accurate and abnormalities were found on the involved side but in one a contralateral collection was missed. In the other two the localization was grossly inaccurate. Eleven cases had post-operative electroencephalograms. Suppression of activity and slow waves, often with large delta foci, were again frequent findings. In four cases the E.E.G. showed abnormalities in the region where pus was extending and these were recordings during a relapse. Once again, intracerebral abscess was usually diagnosed, but the localization was fairly accurate. Seven cases showed an improving post-operative record with return to normal rhythm and areas previously silent showing recovery of activity. Pre-operatively, therefore, electroencephalography was not of great value in diagnosis; it did, however, reveal abnormality and served to give some rough localization. Post-operatively it was rather more useful, serving to indicate progression of the lesion or improvement.

Carotid angiography was performed on 17 patients, in some cases more than once. Thirteen patients had the investigation pre-operatively and in nine it showed evidence of an extracerebral collection. In two cases angiography failed to demonstrate an existing subdural empyema; the presence of a frontal intracerebral abscess in one of these may have obscured the issue. In the remaining two cases the subdural collection was situated in the posterior fossa and in one of these the angiogram showed hydrocephalic stretching of the pericaleosal artery. Nine post-operative angiograms were performed and seven out of these suggested the presence of a new collection. In two cases this was confirmed by subsequent operation and evacuation. The remaining five cases had no exploration despite which clinical improvement occurred. In most of these the site of the presumed collection was alongside the falx. In another case an air encephalogram gave evidence of a similar space-occupying lesion but this was not confirmed by angiography nor by subsequent operation.

The value of angiography is best illustrated by the following case which also demonstrates the problems of management.

CASE 2 F.M. (R.I. 308845), a 39-year-old clerk with a history of chronic sinus infection, developed increasing headache, vomiting, delirium, and dysphasia some three weeks after recovery from a bad cold and 48 hours before admission. An emergency operation to drain pus from the frontal sinus was performed elsewhere, but the followings day, because of the dysphasia and a mild right hemiparesis, he was transferred to this Unit as a case of suspected frontal abscess. The cerebrospinal fluid contained 132 cells per c.mm., mainly lymphocytes, and $56 \mathrm{mg}$. of protein per $100 \mathrm{ml}$. On admission on 1 June 1961 he was alert but aphasic with a total right flaccid hemiplegia. A left carotid angiogram confirmed the diagnosis of a leftsided subdural collection (Fig. 4a). A retrospective examination of the angiograms suggested absent or poor filling of the frontal veins. At operation the same day, a right frontal burr hole revealed no pus, but over the whole left fronto-temporal and anterior parietal regions abundant thick creamy-yellow pus was found. Catheters were inserted for penicillin instillation. Postoperatively, he developed frequent right focal fits, but with general and local administration of penicillin he improved over the next few weeks to almost complete recovery. Then over three or four days he relapsed in=o sidiously with general malaise, drowsiness, and finallyo with a paraparesis most marked in the left leg. Bilatera $N$ carotid angiograms (19 June) revealed evidence of केषे 을 parasagittal collection (Fig. 4b), and bilateral burr holes $\rightarrow$ revealed subdural pus again on the left, but only a few flecks on the right. More catheters were inserted and $\frac{\rho}{0}$ systemic and local antibiotic treatment continued. He failed to regain consciousness after this operation, which. was under general anaesthesia, and required a trache $\frac{0}{5} \vec{\varphi}$ ostomy. For several days he remained comatose, with chest infection and continuous left focal fits. On 23 June, because of his failure to improve, new burr holes were made revealing a small right-sided subdural haematoma and further left frontal pus. His condition still remained desperate, however, and one week later (1 July) new bilateral carotid angiograms demonstrated a further right subdural collection and more right-sided burr holes were made. An extensive subdural abscess was found at the site of the former haematoma, which was becoming encapsulated. Further catheters were inserted and intensive penicillin administration was continued, and within a few hours of operation he showed a striking improvement. He then made a slow recovery from the coma and double hemiplegia. He spoke for the first time since the initial relapse on 17 July, by which time the fits had stopped. Finally, two months after admission, all antibiotic treatment was stopped. He continued to improve over the next two months and was transferred to the Neurological Department at Stoke Mandeville Hospital for rehabilitation. After a few weeks he was mobilized and was discharged home. His improvement continued and at the time of his last attendance in September 1962 he was able to look after himself and to perform his previous occupation of a clerk. He still had focal fits which were becoming less frequent. 


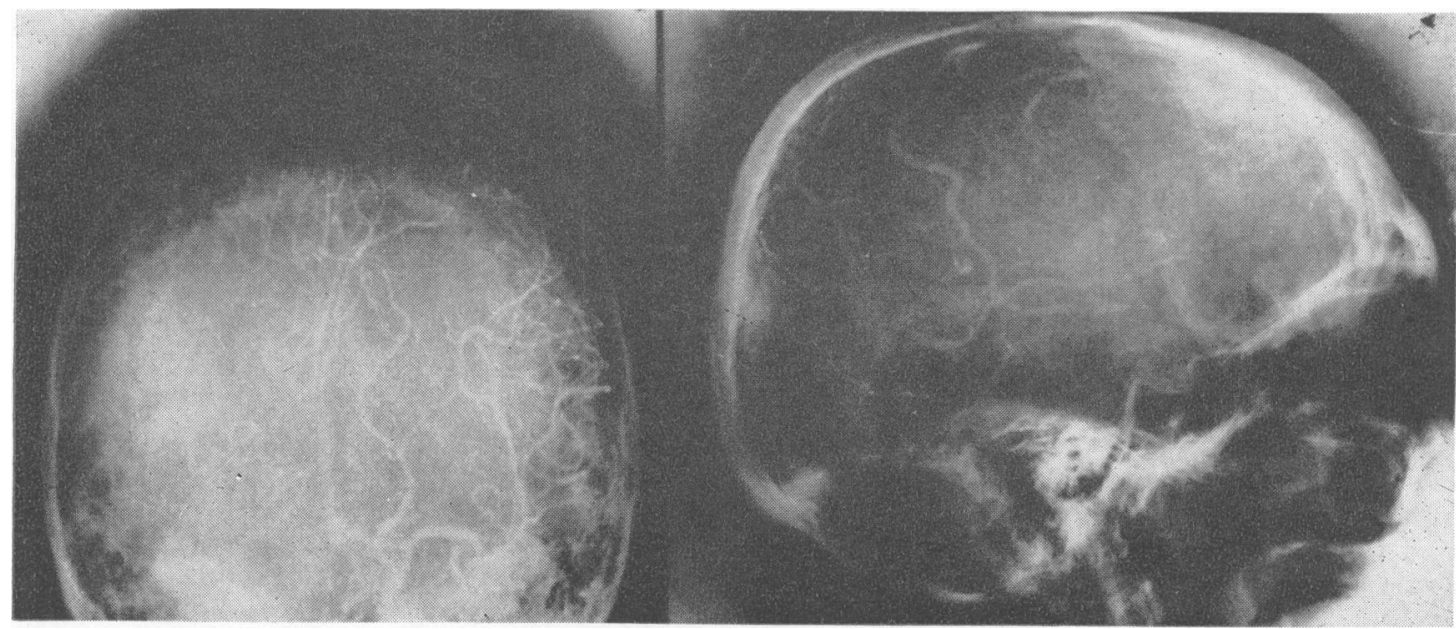

FIG. $4 \mathrm{a}$

FIG. $4 b$

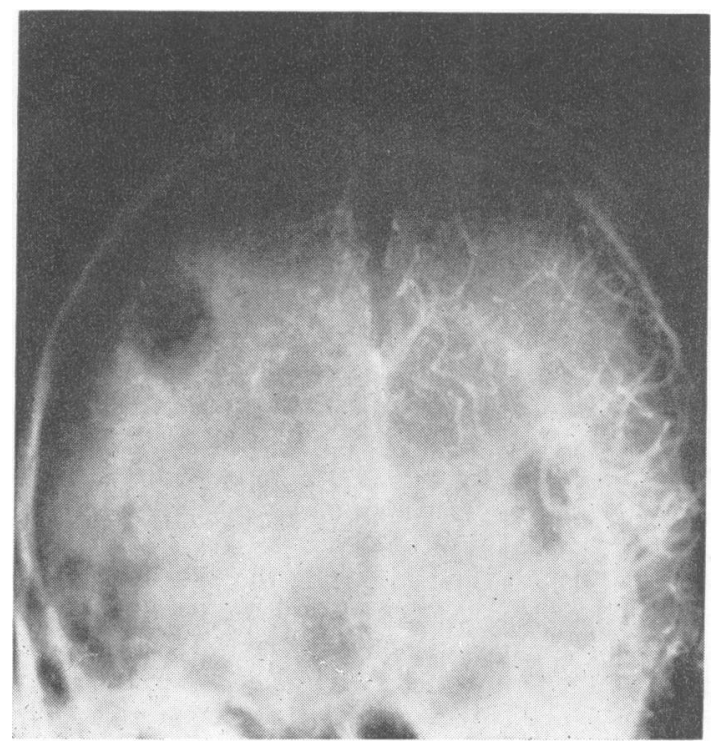

FIG. 4. Case 2. (a) Left carotid angiogram showing displacement of the middle cerebral vessels away from the inner table of the skull in the antero-posterior view. The lateral view of the phlebogram of the same examination shows poor filling of the frontal veins.

(b) Left carotid angiogram showing displacement of the posterior branches of both pericallosal arteries away from the midline.

In our series air encephalograms were rarely performed before operation and only when the diagnosis of subdural empyema was unsuspected. Three cases had pre-operative air studies; one revealed hydrocephalus and two demonstrated displacement of the fourth ventricle (in pre-pontine and posterior fossa purulent collections). Seven cases had air studies performed because of relapse, and all revealed ventricular distortion. Five showed evidence of a parafalcine space-occupying lesion but only two were explored and in these pus was found. In one patient air encephalography suggested the presence of a frontal mass which was subsequently shown to be an intracerebral abscess. In a further case air encephalography merely demonstrated hydrocephalus.
Twelve patients had air studies performed before their discharge in order to exclude any further lesion. Seven of these revealed slight abnormalities such as ventricular dilatation, slight ventricular shift and on the involved side a loss of subarachnoid air (Fig. $5 \mathrm{~b}$ ), presumably because of closure of the subarachnoid space by exudate. This block in the cerebrospinal fluid pathway may account for the common finding of developing papilloedema in cases otherwise improving. In one patient evidence of frontal abscess, previously demonstrated during a period of relapse, was again found but this time markedly increased in size and subsequently this abscess was removed. Two patients were perfectly normal.

Angiography, in our opinion, is the most useful 
special investigation. Its chief value lies in confirming the diagnosis and revealing sites of pus clinically unsuspected and in this it had a high rate of success. It also helps to exclude or demonstrate the presence of an associated intracerebral abscess. In the cases where it failed special views, such as anterior obliques, might well have revealed the collections. However, a negative angiogram does not exclude the presence of a subdural empyema.

If all these special studies are negative the disease once suspected must be excluded by burr hole exploration.

Some patients are dangerously ill on admission and operation is performed immediately as no delay is permissible. In these cases the finding of pus on the convexity of the brain may satisfy the surgeon, but further collections or associated lesions, such as intracerebral abscess, must be excluded and as soon as the patient's condition permits an angiogram should be performed.

\section{TREATMENT}

In this series treatment consisted in making burr holes sited over the suspected area of involvement, but attempts to get to the limits of the collection were rarely made. The pus was removed by depressing the brain, and occasionally the space was irrigated with penicillin. The subdural collection was rarely very large; it was impossible to make accurate measurements but the amount rarely exceeded $20 \mathrm{ml}$. Rubber catheters were then inserted, sometimes two or three into each hole at the sites of pus. It was not always easy to insert the catheters into the space because of the swollen brain and on one occasion intravenous urea had to be used. Two or three millilitres of intrathecal penicillin $(2,000$ units per $\mathrm{ml}$.) was instilled down each tube, which was then closed off with spiggots. Subsequently penicillin was introduced at varying intervals, usually eight-hourly or fourhourly. Little or no drainage of pus occurred, and the purpose of the tubes was to administer the antibiotic; the initial evacuation was usually sufficient. This procedure went on for approximately five to seven days and then the tubes were removed seriatim. There was no trouble with wound sepsis and the burr holes healed quickly. Throughout this period systemic penicillin and sulphonamides were also being given and fits, when present, were controlled by anticonvulsants. General nutrition was maintained by tube feeding if necessary. At operation a swab was taken for bacteriology and when the sensitivity of the organisms was known the antibiotic was continued or changed to a more appropriate one. In the early days we were cautious in instilling new antibiotics into the subdural space, so a few patients were given systemic wide-spectrum antibiotics, whilst penicillin only was continued down the catheters.

\section{POST-OPERATIVE PROGRESS}

The patients' post-operative improvement was often $\bar{c}$ dramatic and sometimes immediate. Systemic 0 evidence of infection and disturbances of consciousness usually quickly improved and the focal signs of hemiparesis, fits, and hemianopia usually showed a marked improvement by the fifth to seventh day. In a few patients the signs of subdural infection re-appeared in the first 24 to 48 hours. From about the seventh day progress was rapid and if treatment had been successful the patients quickly became mobile. At the time of their discharge they were relatively asymptomatic. This in eight out of 18 cases was at approximately four weeks but varied between nine days and 18 weeks from admission. Air encephalography was usually performed before discharging the patient because of the frequency of intracerebral abscess $(17 \%)$. A failure to improve by the fifth or sixth day, or any increase in signs or development of new signs, encouraged special studies (angiography or ventriculography) and usually new burr holes were made. A relapse after an initial improvement was not uncommon and usually $\mathrm{N}$ was due to a re-accumulation of pus on the con $\frac{\rho}{\mathrm{D}}$ 을 vexity of the brain and even more frequently to the $\overrightarrow{-}$ formation of a falcine collection.

The 'falx syndrome' (List, 1955) was presens oै clinically in 11 of the 29 cases (nine paranasal and two otitic), and presented either early in the cours of the disease or more commonly as a cause of $\mathscr{O}$ relapse. These patients had sensory or motor disturbances in one or other lower limb, more marked distally, and involving the proximal muscle groups progressively. Finally, the trunk, the arm, and ultimately the face, were involved. The sparing of the face is such a characteristic feature that it should immediately lead to a suspicion of a falcine collection. Focal fits localized to one leg or spreading to involve the trunk and arm occurred, and sometimes bilateral falcine collections resulted in a profound paraparesis and a rather vague sensory loss. Such a collection is often indicated a few days before the relapse by a failure of the leg to improve at the same rate as the arm on the hemiplegic side. The spread of the pus over the convexity, however, is accompanied by signs so dramatic that a falcine collection may be overlooked. Usually there is increased pyrexia, general malaise, and a deterioration in the level of consciousness, but it was remarkable that falcine collections appeared not to exert such a profound effect as convexity collections in these respects.

There were a few patients with the 'falx syndrome' in whom evacuation of the falcine pus undoubtedly 
contributed to recovery from the relapse, and on one occasion saved a life. In such cases, therefore, fresh burr holes are indicated. If no collection can be demonstrated it may be reasonably assumed that the disorder is due to thrombophlebitis and that the patient will recover. This happened, without any further operation, in a few cases with clinical evidence of the syndrome. Recovery also occurred in some cases in which falcine collections were demonstrated by angiography but no operation was performed for one reason or another. This is a matter of considerable interest and, to our minds may, represent the influence of antibiotics pervading the subdural space, thus limiting the infection to small parafalcine abscesses. Furthermore it indicates that the origin of symptoms must be attributed to a concomitant thrombophlebitis rather than to pus accumulating in the space. Another explanation is that the angio-
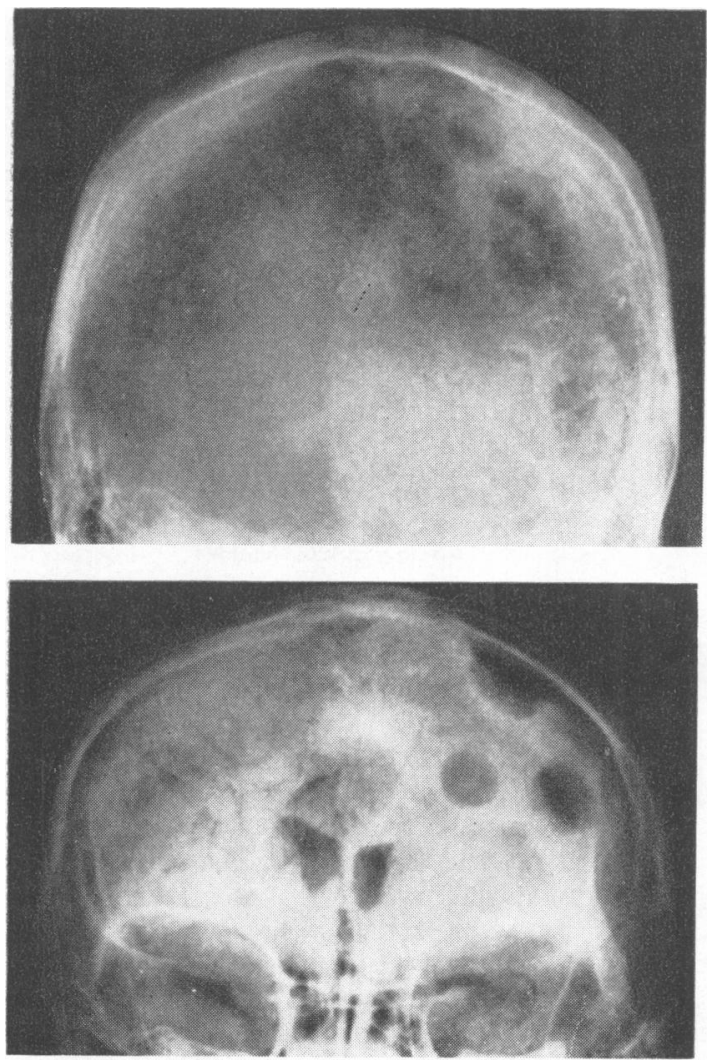

FIG. 5. Case 3. Left carotid angiogram showing displacement of the posterior branches of the pericallosal artery away from the midline.

Air encephalogram showing depression of the left lateral ventricle. It also shows absence of subarachnoid air on the left side. graphic appearances of a collection may be due to localized swelling as a result of thrombophlebitis. The following case illustrates these points.

CASE 3 E.H. (R.I. 138022), a 31-year-old man with a six-week history of frontal sinusitis, suddenly developed weakness of the right leg, progressing to a profound right hemiparesis two days before admission to this unit. $\mathrm{He}$ had been admitted elsewhere and within a few hours became dysphasic, with a pyrexia of $101^{\circ} \mathrm{F}$. The cerebrospinal fluid was cloudy, containing 420 cells per c.mm., largely polymorphs, and $158 \mathrm{mg}$. of protein per $100 \mathrm{ml}$. His condition continued to deteriorate and he was transferred on 28 February 1951. He was found to have a complete right hemiplegia and right homonymous hemianopia, with very slight nominal dysphasia. A left carotid angiogram suggested a falcine collection. Pus was found in the left frontoparietal subdural space and also a small extradural abscess was seen behind the frontal sinus. With systemic and subdural penicillin the power in the right arm improved, but the hemianopia, weakness of the right leg, and cortical sensory loss remained. On 9 March the left carotid angiogram was repeated (Fig. 5a) and the falcine collection appeared to have increased. The following day the leg began to recover and shortly afterwards the hemianopia decreased. On 21 March an air encephalogram (Fig. 5b) demonstrated depression of the left lateral ventricle, regarded as due to a small left parasagittal space-occupying lesion. A falcine exploration was arranged, but before this could be done the right leg weakness greatly improved and the visual fields expanded. The patient was discharged but returned for left carotid angiograms on 16 May by which time the appearances were normal. There had been further improvement in the sensation and power of the right foot. One year later he had given up the toe-raising spring prescribed, and 10 years later (11 April 1962) he had only a trace of weakness in the left foot.

\section{RESULTS OF TREATMENT}

In assessing the results of the treatment, we examined the cases in the light of the rapidity of onset, delay

TABLE V

SENSITIVITY OF ORGANISMS AND PROGRESS IN SUBDURAL INFECTIONS

Subdural and Penicillin and No Subdural Total

Systemic Wide-spectrum Penicillin

Penicillin Antibiotics

Subdurally

\begin{tabular}{lrllr}
\hline Total & 24 & 2 & 3 & 29 \\
Dead & 6 & 1 & 3 & 10 \\
Sensitivity unknown & 10 & 0 & 1 & 11 \\
Dead & 2 & 0 & 1 & 3 \\
Penicillin-sensitive & 9 & 0 & 1 & 10 \\
Dead & 2 & 0 & 1 & 3 \\
Penicillin-resistant & 5 & 0 & 1 & 6 \\
Dead & 2 & 0 & 1 & 3 \\
$\begin{array}{l}\text { Penicillin-resistant } \\
\text { but sensitive to }\end{array}$ & & & & \\
wide-spectrum anti- & & & & \\
biotics & 0 & 2 & 0 & 2 \\
Dead & 0 & 1 & 0 & 1
\end{tabular}


in treatment, bacterial sensitivity, and extent of the collection of pus. The numbers were too small to allow any statistical analysis in these respects and we are only able to give our impressions.

There appeared to be no particular relationship between mortality and the time taken to develop the established condition. On the other hand, there appeared to be some relationship between delay in treating established cases and mortality rate; although two patients who had the infection for 20 and 31 days respectively, survived, in general those who did best were treated early.

A relationship appeared to exist between bacterial sensitivity and morality (Table V). Cases with penicillin-resistant organisms appeared to have a higher mortality rate. In fact bacterial resistance was of greater importance than the extent of the collection of pus. After a trial of penicillin had failed, subsequent treatment with wide-spectrum antibiotics produced no improvement, possibly because their administration was delayed.

ANALYSIS OF DEATHS The overall mortality was 10 out of 29 cases, but three of these were undiagnosed and therefore untreated, so that the mortality rate for treated cases is seven out of 26 cases. Of these, two were admitted moribund and had little hope of recovery. Excluding cases untreated or with delayed diagnosis, the mortality rate was five out of 24 . In two further patients diagnosis after admission to the neurosurgical unit was delayed. One patient died of pulmonary embolus and electrolyte imbalance, but in addition had an undiagnosed falx collection, to which the death may be partially attributed. Two patients died of undiagnosed cerebral abscess.

The association with cerebral abscess formation is interesting. Five of the 29 patients had intracerebral abscess, either concomitantly or subsequently; three of these were from paranasal infection and two from otitic. Of these five patients, three died. The danger of diagnosing subdural empyema, evacuating the collection, and failing to treat an intracerebral abscess is important. Similarly the treatment of intracerebral abscess only, in the presence of a subdural collection of pus may also be fatal.

\section{SUBSEQUENT PROGRESS}

There was a rapid improvement in many of the disabilities until the time of the patient's discharge: hemiplegia, hemianopia, and aphasia may clear up almost completely.

If there was a slight residual disability, e.g., slight weakness of one leg, improvement was observed to occur over a period of three years. A few patients continued to improve after that period, but at the end of three years most patients had little or no을 disability.

Two patients did badly, however. One subse- $-\frac{\bar{Z}}{2}$ quently died in a mental hospital with severe mentalen changes and aphasia, and the other, a child, has hadis to have special schooling, largely because of un- 0 controllable epilepsy.

Epilepsy is a frequent presenting symptom of this? condition and it may persist during the acute stage Of the 19 survivors, $17(89 \%)$ had fits while in? hospital. The incidence of epilepsy thereafter de clines so that at the end of three years the number of patients still having fits had fallen to five $(26 \%)$. However, although epilepsy occurring in the acute stage of the illness may cease when the infection is mastered it may recur in the future, and of the $12^{\circ}$ patients who have been followed up for more thanes three years, four $(33 \%)$ had fits. Two of the survivors $\overrightarrow{0}$ with fits also had intracerebral abscesses treated.

These figures are comparable to those of North- $\vec{\omega}$ croft and Wyke (1957) but the number with fits wills probably increase, as one patient had her first? seizure five years after the infection; she was one of the two who had no fits during the acute stage. There. are no patients in this series who have not suffered seizure either at the onset of the illness or some yeafs later.

This high incidence of epilepsy leads us to adv the administration of anticonvulsants in all cases foro an indefinite period. This is the practice of the de-? partment. In fact all 19 patients had anticonvulsariso while in hospital. However, at the end of the first ${ }^{9}$ three years only 13 of the 19 survivors were stip taking them and thereafter only three.

\section{SUGGESTED MANAGEMENT}

Amongst the methods advised in the treatment of this condition, the most usual is multiple burr holes to allow the frequent instillation of antibiotics and to a lesser extent to provide drainage. Glass (1947)응 and Le Beau (1949), however, advised an osteoplastic flap, and Keith (1949) a limited craniectomy in all cases. In our experience such procedures are rarely necessary and then only if an extensive exploration seems indicated or if there is gross osteomyelitis.. Occasionally an osteoplastic flap is necessary to 3 expose and remove thick exudate.

In all cases, it is important to exclude the presence of bilateral collections of pus, and it is obviously desirable to explore the presumed normal side first? through a clean field. In cases of paranasal infection $>$ the burr hole is sited about $2.5 \mathrm{~cm}$. from the midline in the pre-coronal frontal region, and if no pus is revealed N the wound is closed. A similar frontal burr hole should then be made on the affected side, and if pus is found 
a mid-parietal burr hole, again $2.5 \mathrm{~cm}$. from the midline, should be made. If no pus is revealed at this site, it is unnecessary to make an occipital burr hole, but a temporal one should be made in all cases. These burr holes are usually adequate to define the limits of the collection. Other burr holes may be appropriately sited in the regions where the pus appears to extend. When the limits of the collection have been demonstrated pus is evacuated by depressing the brain with a suitable instrument and allowing the pus to well up into the wound. At this stage, a swab should be taken for culture and sensitivity. It is particularly important to make a smear immediately to determine whether the organisms are Gram-positive or Gram-negative, so that an appropriate antibiotic can be administered. The brain is often swollen and easily damaged, and great care is necessary in depressing the cortex, especially at the margins of the collections. Some authors have advocated lumbar puncture in order to collapse the cerebral hemisphere and so facilitate the introduction of drainage tubes and irrigation of the subdural space. In one case we produced the same effect by administering intravenous urea, but such procedures are usually unnecessary and inadvisable, in that they possibly facilitate further spread. When it appears that all the pus has been evacuated, number 8 or 10 French soft rubber catheters should be inserted through each burr hole. Usually two or three catheters inserted radially in different directions allow the subdural space to be well irrigated with the antibiotic solution.

In the otitic cases, the first burr hole should be made mid-parietal on the healthy side, again $2.5 \mathrm{~cm}$. from the midline, since extension to the opposite hemisphere usually occurs via the falx. On the affected side an occipital parasagittal burr hole is then made and if pus is found a mid-parietal hole as well. Again further burr holes may be made to define the limits of the collection. In all cases, however, a temporal burr hole should be made.

We have used subdural instillations of penicillin solutions as used for intrathecal injections at a strength of approximately 2,000 units per ml., and upon occasion we have also used $50 \mathrm{mg}$. of streptomycin per millilitre. The actual dose of penicillin varied; we have been able to insert a total up to $20 \mathrm{ml}$. of antibiotic solution through the subdural catheters. In patients with epilepsy there may appear to be an increase in seizures during the instillation of penicillin but this need not cause anxiety. Our bacteriological studies have shown that the organisms are frequently penicillin-resistant (see Table I). However, in practice, although laboratory sensitivities are useful, they do not always accord with clinical experience and accordingly we suggest that penicillin and streptomycin should be administered both locally and systematically in all cases at least until the sensitivities become available. Biehl (1955) also suggested this combination because of the predominance of non-haemolytic streptococci in such infections. In the case of otitic infections where proteus organisms are commonly present streptomycin should always be used. We have given erythromycin, $50 \mathrm{mg}$. per ml., into the subdural space without any abnormal reactions, and also tetracycline. These wide-spectrum antibiotics would be effective substitutes for penicillin and streptomycin in cases with resistant organisms. The antibiotic used in the tubes is also given systemically and sulphadiazine and potassium citrate are given by mouth. Subarachnoid therapy is rarely necessary but a lumbar puncture should be performed every three or four days during the acute illness to exclude the development of meningitis. Penicillin may then be injected intrathecally.

The subdural catheters should be spigotted and the burr holes sutured, the tubes being tied to one of the stitches. It is convenient to gather the tubes together with a rubber band, bringing them through the dressing on the head so that subsequent instillations may be given without disturbing the wounds. The antibiotic should be instilled at four-hourly intervals for the first 24 hours and then eight-hourly, and the catheters left in situ for four or five days. Some may become blocked, and if they cannot be unblocked easily by irrigation or shortening of the tube, they should be removed. After two or three days it is a common experience that the volume of fluid it is possible to instil becomes less. As the tubes are not used for drainage they should be spigotted after each instillation. There may be a little release of pus, however, and this should be cultured for organisms and sensitivity. Even after the removal of the tubes systemic antibiotics should be continued for a further seven or eight days in spite of the patient's improvement.

Signs of relapse or failure to improve imply either that the organisms are insensitive to the antibiotic administered, or that a fresh collection has formed inaccessible to the irrigating fluids. Angiography is the most useful investigation and may then reveal a fresh collection which will require more burr holes and tubes. Apart from new collections, however, the subdural empyema may re-accumulate on the same side, and it may then be necessary either to re-open the burr holes or make new ones. If investigations or inspections suggest loculation and formation of a thick capsule, it may be necessary to turn an osteoplastic flap to remove it, but it has not been considered necessary in our series.

The general condition of the patient should receive attention throughout, and especially with 
regard to adequate fluid intake and physiotherapy for paralysed limbs. The relapses are usually insidious but may be acute and these patients require the keenest observation throughout their stay in hospital.

\section{SUMMARY}

The clinical, laboratory, radiological, and pathological features of 29 cases of subdural empyema admitted to the Radcliffe Infirmary from 1949 to 1962 inclusive are reviewed.

Paranasal sinus or ear infection was the usual cause.

Pathways for the extension of pus in the subdural space are described.

The problems of diagnosis are discussed and the value of angiography emphasized.

The occurrence of penicillin-resistant organisms, especially in cases following otitic infections, is stressed, and an operative method of treatment, using multiple burr holes and subdural instillations of penicillin and streptomycin, and on occasion widespectrum antibiotics, is advocated.

We have pleasure in acknowledging the advice and assistance of Mr. Joe Pennybacker in the preparation of this report of patients passing through the Department of Neurological Surgety Mr. John Potter kindly read the manuscript and made helpful suggestions. Dr. Phil Sheldon advised us on the radiology. This work w undertaken while E.H. was in receipt of a grant from the Sir Hugh Cairns Memorial Fund.

REFERENCES

Biehl, J. P. (1955). Subdural empyema secondary to acute front sinusitis. J. Amer. med. Ass., 158, 721-724.

Courville, C. B. (1944). Subdural empyema secondary to purulent frontal sinusitis. Arch. Otolaryng., 39, 211-230.

Glass, R. L. (1947). Osteoplastic flap method in the treatment subdural abscess. $J$. Neurosurg., 4, 391-393.

Keith, W. S. (1949). Subdural empyema. Ibid., 6, 127-139.

Kubik, C. S., and Adams, R. D. (1943). Subdural empyema. Brain, $18-42$.

Le Beau, J. (1949). Traitement chirurgical de l'empyème sous-dur et sous-arachnoidien. Rev. neurol., 81, 828-851.

List, C. F. (1955). Diagnosis and treatment of acute subdural erou

pyema. Neurology (Minneap.), 5, 663-670.
Northcroft, G. B., and Wyke, B. D. (1957). Seizures following surgic $\vec{P}$ treatment of intracranial abscesses, J. Neurosurg., 14, 249-263:-

Schiller, F., Cairns, H., and Russell, D. S. (1948). The treatment purulent pachymeningitis and subdural suppuration with special reference to penicillin. J. Neurol. Neurosurg. Psychiat 11, 143-182.

Stern, W. E., and Boldrey, E. (1952). Subdural purulent collection Surg. Gynec. Obstet. 95, 623-630.

Stephens, J., and Welch, K. (1956). Subdural abscess with retina thrombophlebitis, Neurology, 6, 889-890.

Wood, P. H. (1952), Diffuse subdural suppuration. J. Laryng 496-515. 